www.scielo.cl

\title{
Necrosis grasa del recién nacido
}

\author{
Fat necrosis of the newborn
}

\section{Daniel López Hurtado ${ }^{a}$, Mario Elías Delgado ${ }^{\mathrm{b}}$, Javier Ortega Díaz ${ }^{\mathrm{b}}$, Marco Solís Avaca $^{\mathrm{b}}$, Heyssel Carmona Alvaradob ${ }^{b}$ Cecilia Cabello Durán ${ }^{c}$}

\author{
aUnidad de Neonatología, Servicio de Pediatría, Hospital San Juan de Dios de Curicó, Chile

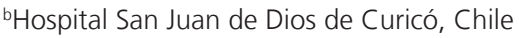 \\ 'Estudiante de Medicina, Facultad de Ciencias Médicas, Universidad de Talca, Chile
}

Recibido: 29 de marzo de 2019; Aceptado: 3 de agosto de 2019

¿Qué se sabe del tema que trata este estudio?

La necrosis grasa del recién nacido (NGRN) es un trastorno poco común caracterizado por nódulos y placas eritematosas, por lo general en los recién nacidos a termino. Frecuentemente sigue un curso autolimitado, pero puede complicarse con hipercalcemia, entre otras.

\section{¿Qué aporta este estudio a lo ya conocido?}

Presentamos un paciente portador de una anomalía de Epstein que podría representar un factor de riesgo para el desarrollo de NGRN, un aporte original dado que son otras las cardiopatías congénitas descritas en la literatura asociadas a NGRN.

\section{Resumen}

Introducción: Las paniculitis constituyen un grupo de enfermedades que afectan al tejido graso subcutáneo y se manifiestan clínicamente como nódulos. Su patogenia no es del todo clara, por lo general es asintomático. El diagnóstico confirmatorio es histológico. Objetivo: describir las características clínicas e histopatológicas de un caso de necrosis grasa, una forma especifica de paniculitis en el recién nacido $(\mathrm{RN})$. Caso Clínico: $\mathrm{RN}$ femenino de 40 semanas, parto cesárea de urgencia por taquicardia fetal más meconio, APGAR 7-8-9. Requirió oxigeno y presión positiva por 5 min. Al quinto día de vida presentó aumento de volumen en tronco posterior, de coloración eritematosa - violácea, remitente a la palpación, no doloroso. Ecotomografría de piel y partes blandas informó aumento de la ecogenecidad del tejido celular subcutáneo y pérdida de definición de las celdillas adiposas de aproximadamente 42.3 x 9.7 x $20.1 \mathrm{~mm}$, sin presencia de vascularización. Biopsia de piel: epidermis con acantosis irregular y ortoqueratosis en cestas; dermis papilar con infiltrado inflamatorio y dermis reticular y tejido adiposo con presencia de infiltrado linfohistocitiario con tendencia a la formación de nódulos, sin compromiso vascular, leves depósitos de colesterol; compatible con necrosis grasa del recién nacido (NGRN). Paciente tuvo regresión completa de la lesión a los tres meses de vida. Conclusiones: se describe un caso compatible clínica e histológicamente con NGRN; sin complicaciones durante su observación. Por lo general esta patología tiene buen pronóstico, con resolución espontánea, como en nuestro caso.
Palabras clave: paniculitis; necrosis grasa; recién nacido; neonatología 


\begin{abstract}
Introduction: Panniculitis is a group of diseases that affect subcutaneous fat tissue and clinically manifest as nodules. Its pathogenesis is not entirely clear, and it is usually asymptomatic. The confirmatory diagnosis is histological. Objective: To describe the clinical and histopathological characteristics of a case of fat necrosis, a specific form of panniculitis in the newborn (NB). Clinical Case: 40-week female NB, born by emergency cesarean section due to fetal tachycardia with meconium, Apgar score 7-8-9. She required oxygen and positive pressure for five minutes. On the fifth day of life, she presented an increased volume in the posterior trunk region, with an erythematous - purplish discoloration, which is soft and non-tender to palpation. Skin and soft tissues ultrasound showed increased echogenicity of the subcutaneous cellular tissue and loss of definition of the adipocytes of $42.3 \times 9.7 \times 20.1$ $\mathrm{mm}$ approximately, without vascularization. Skin biopsy showed epidermis with irregular acanthosis and basket-weave orthokeratosis; papillary dermis with inflammatory infiltrate, and reticular dermis and adipose tissue with presence of lymphohistiocytic infiltrate with a tendency to form nodules, without vascular involvement, and small cholesterol deposits, compatible with subcutaneous fat necrosis (SBFN) of the newborn. The patient at three months of age had complete regression of the lesion. Conclusions: a clinically and histologically compatible case with SBFN is described, that did not present complications during observation. In general, this pathology has a good prognosis, with spontaneous resolution as in our case.
\end{abstract}

\section{Introducción}

Las paniculitis constituyen un grupo de enfermedades que afectan al tejido graso subcutáneo de etiología muy variada. Pueden ser idiopáticas o asociarse a diferentes enfermedades metabólicas, autoinmunes, neoplasias, fármacos, agentes físicos o infecciosos ${ }^{1}$.

Se manifiestan clínicamente como nódulos en el tejido subcutáneo. La edad del paciente y la ubicación de las lesiones pueden ayudar en el diagnóstico clínico, pero la histopatología es importante para el diagnóstico confirmatorio. Se clasifican de acuerdo a la afectación de la hipodermis, en septales y lobulillares (tabla 1). Existen paniculitis específicas en la infancia como son la necrosis grasa del recién nacido, esclerema neonatorum, post esteroide y por frío; las cuales histológicamente se encuentran dentro del patrón lobar².

El objetivo de este manuscrito es describir las características clínicas e histopatológicas de un caso de NGRN, una forma específica de paniculitis en el RN, poco frecuente en la práctica clínica.

\section{Caso Clínico}

RN de 40 semanas, sexo femenino, acorde para la edad gestacional, producto de embarazo controlado, peso de nacimiento $3.655 \mathrm{~g}$ y longitud $50 \mathrm{~cm}$. Hijo de Rh (-) no sensibilizada. Parto cesárea de urgencia por taquicardia fetal más meconio, APGAR 7-8-9. Requirió oxigeno y presión positiva por $5 \mathrm{~min}$. Se aspiraron secreciones tipo meconio fluido. Sin hallazgos físicos de dismorfias. Test de Coombs directo del RN fue ne-
Keywords:

panniculitis; fat necrosis; newborn; neonatology gativo, y gasometría venosa de cordón umbilical fue normal.

Ingresó a unidad de cuidados intermedios de neonatología por antecedente de depresión neonatal y distres respiratorio (solo taquipnea y estertores bilaterales a la auscultación pulmonar); requirió oxigeno ambiental por $12 \mathrm{~h}$ y luego por naricera hasta las $21 \mathrm{~h}$ de vida, con Rx de Tórax normal; evolucionó luego de las $24 \mathrm{~h}$ de vida sin signos de dificultad respiratoria. El hemograma antes de las $24 \mathrm{~h}$ de vida presentó leucopenia, proteína $\mathrm{C}$ reactiva $(\mathrm{PCR})$ y procalcitonina $(\mathrm{PCT})$ estaban alteradas, por lo que se inició antibioticoterapia empírica por sospecha de sepsis neonatal con Ampicilina y Amikacina por 7 días, con previa toma hemocultivos que resultaron negativos.

Se pesquisó al segundo día de vida soplo sistólico III/VI por lo que fue evaluada por cardiología infantil y se realizó ecocardiograma concluyendo anomalía de Epstein tipo B de Carpentier, comunicación interventricular muscular medio basal, insuficiencia tricuspídea leve y foramen oval permeable, sin signos de hipertensión pulmonar.

Al quinto día de vida presentó aumento de volumen en línea media de tronco posterior, tipo múltiples nódulos eritematosos, indurado, fijos a planos profundos, de $5 \mathrm{~cm}$ en su diámetro mayor cuando confluyen y aparentemente no doloroso al tacto (figura 1). Se realizó control de hemograma, PCR, PCT, tiempos de coagulación, glicemia y calcio dentro de límites normales. Ecografía de piel y partes blandas mostró un aumento de la ecogenecidad del tejido celular subcutáneo con pérdida de la definición de las celdillas adiposas de aproximadamente 42,3 x 9,7 x 20,1 mm que a la señal Doppler color no mostró alteraciones evidencia- 


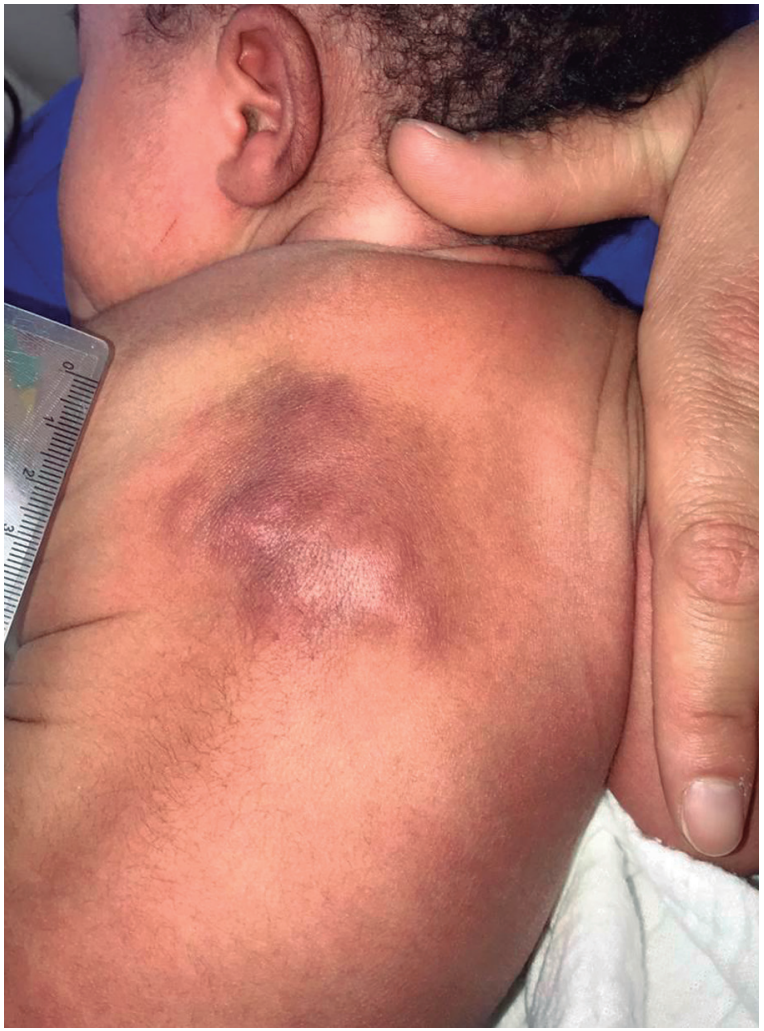

Figura 1. Recién nacido con lesión de aspecto nodular confluyentes, eritematosos en tronco posterior

\begin{tabular}{ll}
$\begin{array}{l}\text { Tabla 1. Paniculitis en pediatría: patrón de inflamación lobular } \\
\text { y septal }\end{array}$ \\
\hline Paniculitis predominantemente & $\begin{array}{l}\text { Paniculitis predominantemente } \\
\text { septal }\end{array}$ \\
lobar & $\begin{array}{l}\text { Eritema nodoso } \\
\text { Paniculitis granulomatosa }\end{array}$ \\
\hline Necrosis grasa subcutánea del & Granuloma anular subcutáneo \\
recién nacido & Poliarteritis nodosa cutánea \\
Esclerema neonataruma & \\
Paniculitis post esteroide & \\
Físico & \\
Paniculitis por frío & \\
Inyecciones & \\
Trauma & \\
Paniculitis infecciosa & \\
Fúngica & \\
Bacteriana & \\
Eritema induratum De Bazin & \\
Paniculitis enzimática & \\
Deficiencia de alfa-1 antitripsina & \\
Paniculitis pancreática & \\
Paniculitis granulomatosa & \\
Sarcoidosis subcutánea & \\
Enfermedad del tejido conectivo & \\
Paniculitis lúpica & \\
Paniculitis en dermatomiositis & \\
Paniculitis histiocítica citofágica & \\
Linfoma de células T paniculítico & \\
subcutáneo & \\
\hline
\end{tabular}

aPanicilutis específica en pediatría. b No es una verdadera paniculitis
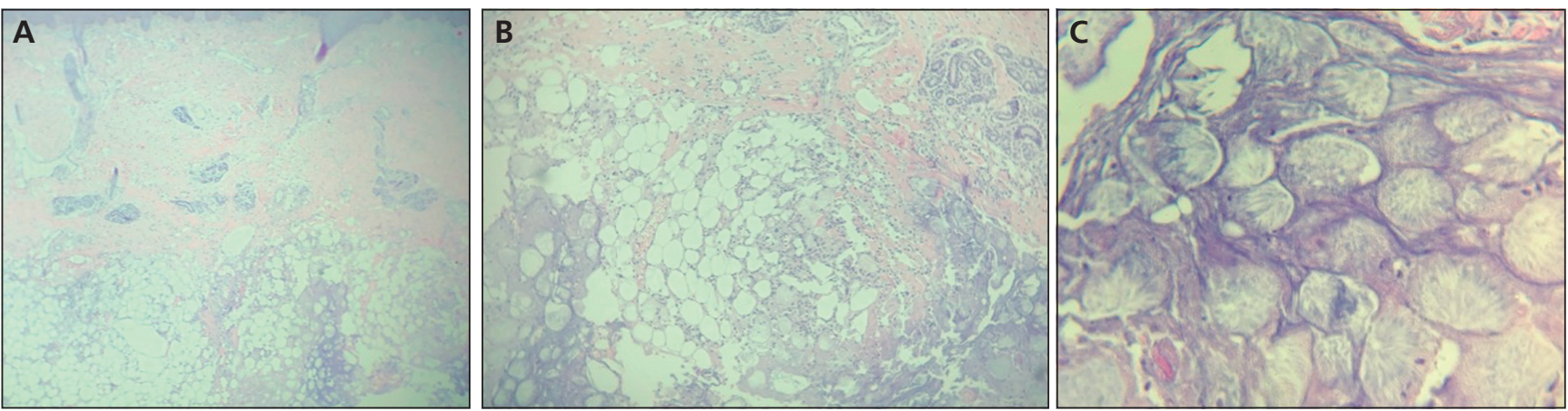

Figura 2. Histopatología - Hematoxilina y Eosina. (A) Aumento 4x: en dermis profunda se observa paniculitis lobular sin presencia de vasculitis. (B) Aumento 10x: se aprecia abundante infiltrado inflamatorio linfohistiocitario con disposición nodular, en las células adipociticas, y presencia de algunas células gigantes multinucleadas. (C) Aumento 40x: cúmulo de cristales lipídicos intracitoplasmático y adipocitico, de disposición radial, sugestivo de necrosis grasa.

bles en la vascularización. Biopsia de la lesión reportó epidermis con acantosis irregular y ortoqueratosis en cestas; dermis papilar con infiltrado inflamatorio y dermis reticular y tejido adiposo con presencia de infiltrado linfohistocitiario con tendencia a la formación de nódulos, sin compromiso vascular, leves depósitos de colesterol; se informó que hallazgos morfológicos son compatibles con necrosis grasa del recién nacido (figura 2). Además se tomó de la lesión en piel: cultivo aerobio, gram, cultivo para $\mathrm{KOCH}$ los que fueron negativos. Se concluyó diagnóstico de necrosis grasa del recién nacido, se mantuvo con controles ambulatorios con pediatría, cardiología y dermatología. Al mes de vida inició regresión de la lesión hasta que desapareció al tercer mes de vida. Calcio sérico controlado hasta el cuarto mes de vida fue normal para la edad. 


\section{Discusión}

La NGRN es una paniculitis benigna cuyo inicio puede variar a lo largo del período neonatal ${ }^{3}$. Es una afección poco frecuente y se desconoce su incidencia precisa. Del Pozzo-Magaña y Nhung $\mathrm{Ho}^{4}$ en un estudio retrospectivo de 20 años sobre NGRN informan una proporción casi igual de hombres a mujeres $(1,14: 1)^{4}$. Con mayor frecuencia se desarrolla en RN a término (más de 37 semanas de edad gestacional) que han experimentado hipoxia u otro estrés perinatal. Entre otros factores de riesgo se encuentran: a) Neonatales: la hipotermia terapéutica para la asfixia ${ }^{5}$, aspiración de meconio, prolapso de cordón umbilical, sepsis, perforación intestinal y cardiopatías congénitas $^{6}$; b) Maternos: preeclampsia, diabetes gestacional, uso de sustancias (bloqueadores de los canales de calcio, cocaína), consumo y/o exposición pasiva de tabaco, incompatibilidad Rh materno-fetal ${ }^{6,7}$. En nuestro caso el paciente presentó una cardiopatía congénita tipo anomalía de Epstein, sin embargo con este tipo especifico de patología no se encontró asociación en la literatura.

La patogenia de la NGRN aún no esta clara; una hipótesis propone que podría ser resultado de la combinación de hipoxia tisular local y presión mecánica; otra sugiere que el enriquecimiento de los ácidos esteárico y los ácidos palmíticos saturados en la grasa neonatal predispone al tejido a la cristalización a bajas temperaturas.

Clínicamente se caracteriza por múltiples placas o nódulos indurados, con o sin eritema en las mejillas, tronco posterior, glúteos y extremidades; mientras que tronco anterior y abdomen por lo general no están comprometidos $^{3}$. Nuestro paciente desarrolló dentro de los primeros días de vida nódulos subcutáneos y eritema en tronco posterior. Del Pozzo-Magaña y Nhung Ho, reportan que los nódulos y eritema están presente en el 100 y $73 \%$ respectivamente en los casos de NGRN, así mismo, la espalda ocupa el 50\% de los RN afectados ${ }^{4}$.

El diagnóstico de NGRN se puede realizar clínicamente, sin embargo, la confirmación es histopatológica; cuyos hallazgos incluyen: paniculitis lobular con un denso infiltrado de histiocitos, eosinófilos y células gigantes multinucleadas; espacios de hendidura de cristales radiales en matrices dentro de adipocitos; calcificación focal dentro de las lesiones; sin vasculitis ${ }^{8}$. Hallazgos compatibles con el resultado de la biopsia de piel del RN presentado. Si existe duda, la resonancia magnética y la ecografía también pueden ser útiles en la evaluación de las lesiones ${ }^{8,9}$.

La resolución de la mayoría de las lesiones ocurre de forma espontánea en semanas a meses sin dejar secuelas en piel $^{9}$, y en algunos casos, la grasa afectada se licua en las ampollas fluctuantes o también puede desarrollarse atrofia cutánea ${ }^{10}$. Nuestro paciente inició regresión de la lesión desde el mes de vida hasta que desapareció por completo a los tres meses de vida. Mismo tiempo de inicio de regresión de la lesión que describen Avayú E ${ }^{11}$. El dolor ocurre en aproximadamente el $25 \%$ de los casos y requiere un tratamiento analgésico apropiado ${ }^{3}$. La NGRN suele ser una condición transitoria y autolimitada, sin embargo, pueden presentar complicaciones como hipercalcemia, trombocitopenia, hipoglucemia e hipertrigliceridemia ${ }^{4}$. La hipercalcemia ocurre en el 36 a 56\% de los neonatos afectados $^{12}$; podría ser grave y/o mortal; se desconoce la etiología específica, sin embargo es probable que células inflamatorias granulomatosas expresen altos niveles de 1-alfa-hidroxilasa, la enzima que convierte 25-hidroxivitamina D3 en su forma activa de 1,25-dihidroxivitamina D3 y ésta última promueva la movilización de calcio del hueso y aumenta su absorción intestinal ${ }^{13}$. Puede ser asintomática o presentar irritabilidad, hipotonía, anorexia o vómitos. La nefrocalcinosis y la calcificación metastásica en otros tejidos (pericardio y cerebro) se han visto asociadas en neonatos con necrosis grasa ${ }^{14,15}$. Por lo tanto los niveles de calcio sérico deben controlarse hasta seis meses después del inicio de las lesiones cutáneas. Se trata con restricción de suplementos de calcio y vitamina $\mathrm{D}$, hiperhidratación con líquidos intravenosos y diuréticos de asa y en casos más graves, se han utilizado corticosteroides sistémicos y bifosfonatos ${ }^{3,16}$. En nuestro caso no se evidenció ninguna de las complicaciones descritas, y en los controles ambulatorios mensuales el calcio sérico fue normal en los 4 primeros meses de vida.

Los diagnósticos diferenciales de la NGRN además de otras paniculitis del RN (esclerema neonatorum (EN), post esteroide y por frío) se encuentran: hemangioma, celulitis, histiocitosis, enfermedad de Faber, fibromatosis y rabdomiosarcomas que son histológicamente diferentes ${ }^{17}$.

Con respecto al EN entre los factores de riesgo descritos están: RN muy graves, prematuros con bajo peso al nacer, sepsis, cardiopatías congénitas, entre otras ${ }^{18}$. Se asocia con una alta tasa de mortalidad, alrededor del $75 \%{ }^{19}$. Las lesiones aparecen los primeros días de vida, inicialmente en glúteos y los muslos, pero se extienden rápidamente para abarcar grandes áreas del cuerpo ${ }^{18}$, sin comprometer los genitales, las palmas de las manos y las plantas de los pies, que carecen de tejido adiposo subcutáneo. La piel endurecida puede aparecer cerosa, morada o moteada. Puede existir limitación funcional de las articulaciones, alteración del movimiento de la pared torácica y dificultad para la alimentación. La histopatología muestra hendiduras en forma de aguja dentro de los adipocitos, pero la falta de células infla- 
matorias lo diferencia de $\mathrm{NGRN}^{18}$. El tratamiento de la EN se dirige principalmente a la enfermedad subyacente $^{19}$.

La paniculitis post esteroides, se presenta debido a altas dosis de este fármaco, por vía sistémica, ya sean orales o intravenosos, seguidos de una rápida retirada de la medicación. De uno a diez días después del cese de los corticosteroides se desarrollan los nódulos. Los hallazgos histopatológicos pueden ser idénticos a los de la NGRN $^{2}$.

La paniculitis por frío se presenta 48 a 72 h después de la exposición a muy bajas temperaturas. Las secciones de tejido muestran una paniculitis en su mayoría lobular con un infiltrado de linfocitos e histiocitos, y un infiltrado dérmico perivascular superficial y profundo sin vasculitis ${ }^{2}$.

\section{Conclusiones}

La NGRN es una patología que de no presentar complicaciones es benigna y autolimitada, sin necesidad de tratamiento. Es importante vigilar a los recién nacidos con especial referencia a la hipercalcemia. Se recomienda el control regular del calcio sérico hasta la edad de 6 meses. Estudios prospectivos permitirían determinar la prevalencia y la incidencia de complicaciones de la NGRN.

\section{Responsabilidades Éticas}

Protección de personas y animales: Los autores declaran que los procedimientos seguidos se conformaron a las normas éticas del comité de experimentación humana responsable y de acuerdo con la Asociación Médica Mundial y la Declaración de Helsinki.

Confidencialidad de los datos: Los autores declaran que han seguido los protocolos de su centro de trabajo sobre la publicación de datos de pacientes.

Derecho a la privacidad y consentimiento informado: Los autores han obtenido el consentimiento informado de los padres (tutores) de la paciente y/o sujetos referidos en el artículo. Este documento obra en poder del autor de correspondencia

\section{Conflicto de intereses}

Los autores declaran no tener conflicto de intereses.

\section{Referencias}

1. Blázquez M, Revenga M, Llop M. Protocolo diagnóstico de las paniculitis. Medicine 2017;12:1599-603

2. Polcari I, Stein S. Panniculitis in childhooddth. Dermatologic Therapy 2010 [cited 2019;23:356-67].

3. Borgia F, De Pasquale L, Cacace C, Meo P, Guarneri C, Cannavo SP. Subcutaneous fat necrosis of the newborn: be aware of hypercalcaemia. J Paediatr Child Health 2006 ; 42:316-8.

4. Del Pozzo-Magaña B, Ho N Subcutaneous Fat Necrosis of the Newborn: A 20-Year Retrospective Study. Pediatr Dermatol 2016;33:e353-5.

5. Hogeling M, Meddles K, Berk D, et al. Extensive subcutaneous fat necrosis of the newborn associated with therapeutic hypothermia. Pediatr Dermatol. 2012;29:59-63.

6. Thomas JM, Bhandari J, Rytina E, Gass JK, Williams RM, Burrows NP. Subcutaneous Fat Necrosis of the Neonate with a Delayed Second Eruption. Pediatr Dermatol 2016;33:e134-6.

7. Rubin G, Spagnut G, Morandi F, Valerio E, Cutrone M. Subcutaneous fat necrosis of the newborn. Clinical Case Reports 2015;3:1017-20.

8. Mahé E, De Prost Y. Subcutaneous fat necrosis of the newborn. Ann Dermatol Venereol 2007; 134:494-8.

9. Kruse K, Irle U, Uhlig R. Elevated 1,25-dihydroxyvitamin D serum concentrations in infants with subcutaneous fat necrosis. J Pediatr 1993;122:460-3.

10. Shumer D, Thaker V, Taylor G, Wassner AJ. Severe hypercalcaemia due to subcutaneous fat necrosis: presentation, management and complications. Arch Dis Child Fetal Neonatal 2014; 99:19-21.

11. Vayú E, Rodríguez C, Wortsman X, Corredoira Y, Serman D, Strauch G. et al. Necrosis grasa del recién nacido: A propósito de un caso. Rev Chil Pediatr 2009;80:60-4.

12. Mahé E, Girszyn N, Hadj-Rabia $S$, Bodemer C, Hamel-Teillac D, De Prost Y. Subcutaneous fat necrosis of the newborn: a systematic evaluation of risk factors, clinical manifestations, complications and outcome of 16 children. Br J Dermatol 2007;156:709-15.

13. Beuzeboc G, Aillet S, Bertheuil N, Delliere V, Thienot S, Watier E. Surgical management of subcutaneous fat necrosis of the newborn required due to a lack of improvement: a very rare case. $\mathrm{Br} \mathrm{J}$ Dermatol 2014;171:183-5.

14. Akin M, Akin L, Coban D, Akcakus M, Balkanli S, Kurtoglu S. Post-operative subcutaneous fat necrosis in a newborn: a case report. Fetal Pediatr Pathol 2011;30:363-9.

15. Torrelo A, Hernández A. Panniculitis in Children. Dermatol Clin 2008; 26:491500.

16. Hunt R. Subcutaneous fat necrosis of the newborn. In: Levy M, Corona R, editors. UptoDate. 2019; Jan 05.

17. Lara L, Villa A, Otero M, Akcakus M, Balkanli S, Kurtoglu S. Subcutaneous Fat Necrosis of the Newborn: Report of Five Cases. Pediatrics and Neonatology 2017;58:85-8

18. Nair S, Nair S, Borade A, Ramakrishnan K. Hypercalcemia and metastatic calcification in a neonate with subcutaneous fat necrosis. Indian J Pediatr 2009;76:1155-7.

19. Weedon D. Chapter 17, Paniculitis en Weedon's Skin Pathology London: Churchill Livingstone Elsevier; 2016. p. 532-55. 